\title{
Oncovascular Surgery-The Multidisciplinary Approach: Surgical Resection of the Musculoskeletal Tumor and Associated Revascularization
}

\section{Ascoli Marchetti Andrea ${ }^{*}$, Di Giulio Lorenzo, Citoni Gianluca, Ippoliti Arnaldo}

Vascular Surgery Unit, Biomedicine and Prevention Department, University of Rome Tor Vergata, Rome, Italy

*Corresponding Author: Ascoli Marchetti A, Vascular Surgery Unit, Biomedicine and Prevention Department, University of Rome Tor Vergata, Rome, Italy, E-mail: ascolimarchetti@med.uniroma2.it

Received: 10 August 2019; Accepted: 26 August 2019; Published: 30 August 2019

\begin{abstract}
Introduction: The possibility of preserving the limb during the definitive removal operation in the case of sarcoma is a goal pursued in a multidisciplinary perspective. The aim of the study is to report our experience gained in collaboration with the IFO oncology orthopedic division and with the operating units of Orthopedics A and B of the Tor Vergata Policlinico, reporting the results and complications of the procedures performed.
\end{abstract}

Methods: From 2011 to 2014, 30 patients treated had primitive and secondary musculoskeletal neoplasm with a mean age of 44 years (14-67 years) in 26 cases (86.6\%) were required revascularization. Women accounted for $53.84 \%$ (14/26), $46.16 \%$ were men (12/26). The preoperative study included a biopsy of the lesion and ultrasonography and angio-CT with multiplanar reconstructions for the evaluation of the vascular anatomy and neoplasia extension.

Results: The average postoperative follow-up was 19.4 months. The reconstruction of the vessels has been implemented in 12/26 (46\%) mainly using the autologous saphenous contralateral vein. The use of the prosthesis was performed in the others cases (with PTFE and Dacron K prosthesis). In 4 cases the patients had lower limb edema, with a distance venous patency of $80 \%$ and arterial patency of $100 \%$. We had no mortality at 30 days. Two patients underwent VAC therapy. No amputations had at 30 days. In 14 patients were perform chemotherapy or radiotherapy before and after surgery.

Conclusions: A long-term evaluation is needed to determine the implications at distance. In case of involvement of 
arterial and venous vessels, vascular surgery skills allow radical resection and function preservation. The mortality gap depends by the prognosis of neoplasm.

Keywords: Sarcoma; Limbs sparing; Artery reconstruction; Vein reconstruction

\section{Introduction}

The vascular management of patients affected by resectable neoplasm provides the use of principles of oncovascular surgery in operative planning particularly when preparing for a ligation with complete removal or when performing an arterial or venous reconstruction. In patients affected by lower limb soft tissue sarcomas, the involvement of the neurovascular structures is $3 \%$. Although is a rare occurrence, it requires vascular surgery [1].

\section{Methods}

In a four years period from 2011 to 2014, in our center were treated 30 patients affected by primitive and secondary musculoskeletal neoplasm with a mean age of 44 years (14-67 years) in 26 cases (86.6\%) were required revascularization. Women accounted for $53.84 \%$ (14/26), $46.16 \%$ were men (12/26). The preoperative study included a biopsy of the lesion and ultrasonography and angio-CT with multiplanar reconstructions for the evaluation of the vascular anatomy, neoplasia extension and a consequent accurate planning of the intervention after oncological, orthopedic and vascular surgery opinion.

\section{Results}

An average postoperative follow-up of 19.4 months were performed. 12/26 (46\%) patients have been treated by autologous saphenous contralateral vein. The use of the prosthesis was performed in 14/26 (PTFE and Dacron K prosthesis). 4 patients developed lower limb edema, associated with venous patency of $80 \%$ and arterial patency of $100 \%$ at long term follow up. At 30 days, the survival rate was $100 \% \cdot 14 / 30$ patients have been treated with perioperative chemotherapy or radiotherapy.

\section{Discussion}

Fortner described the first case of vascular reconstruction in a patient affected by lower limb sarcoma in 1977 [2]. In the following years, the concept of limbs preserving surgery has been progressively adopted, when possible, for the improvement of the quality of life. This objective can be achieved with a collaboration between all the specialists involved in the decision-making process. The role of multidisciplinary team, including oncologic, orthopedic and vascular surgeons, is necessary in patients affected by lower limbs soft tissue sarcoma. In particular, a preoperative planning and cooperation in the postoperative period are crucial [3]. Lower limb soft tissue sarcoma can be rarely removed preserving the arterial and venous vessels. Major vascular resection and reconstruction are required for adequate oncologic margins. The surgical excision of the neoplasm associated with artery and/or vein reconstruction has been reported. A mortality rates of $0-4.8 \%$ has been reported in previous case series. $86-100 \%$ was the tumor control rate and $92-94.1 \%$ was the limb salvage rate. 
Following the surgery, edema was clinically evident in about $40 \%$ of the treated cases, no difference has been observed between the two different treatments respectively veins treated with grafting or with ligation as Schwarzbach and Tsukushi $[4,5]$. In our experience 4/26 patients presented postoperative edema with the same characteristics. 33-57\% of the patients developed wound complications, associated with dehiscence reported. The wound complications has been succesfully treated with VAC therapy. The graft infection rates of 6-29\% have been reported in literature [4, 8-11]. No infections were observed in our experience Table 1. A multidisciplinary setting is crucial for the radicality of the surgery [12-15] (Figures 1-2).

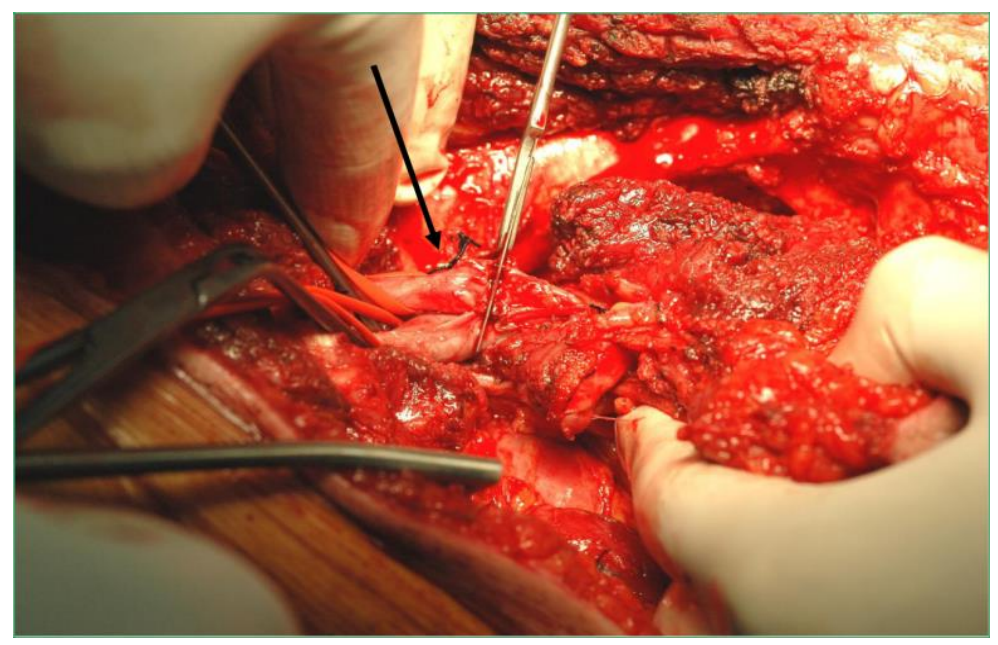

Figure 1: Intraoperative image. The scalpel resets the neoplasia proximally. The non involved vessels are clamped. The arrow indicates the superficial femoral artery and vein to the left of the scalpel.

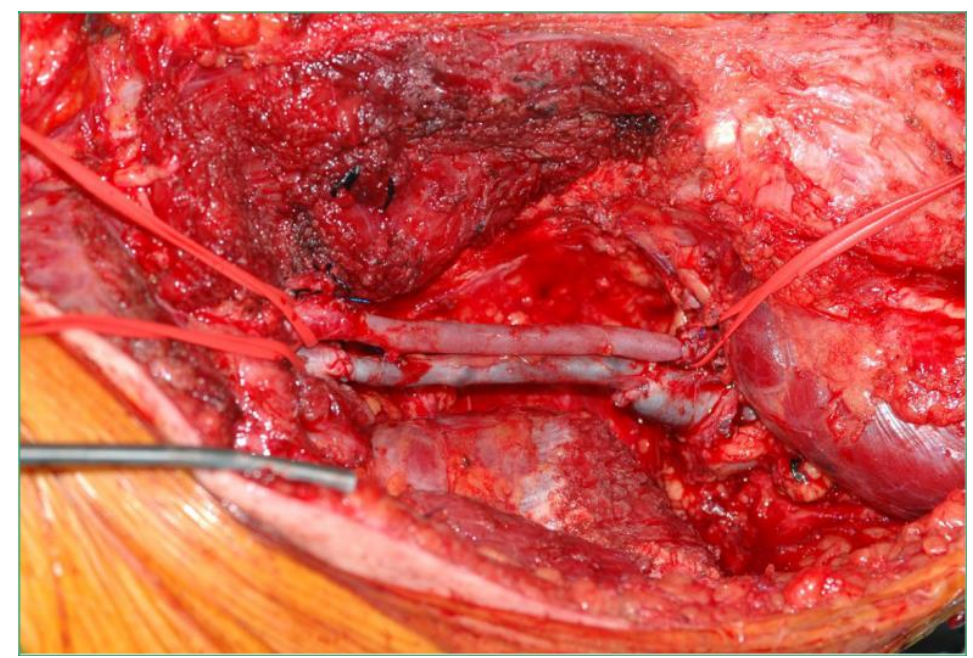

Figure 2: Intraoperative image. The superficial femoral artery and the superficial femoral vein are removed with the sarcoma. A IGVS (inverted great safenous vein) has been grafted to replace the removed artery tract. The VSA (great safenous vein) has been grafted to replace the removed vein tract. 
In particular cases the removal of the neoplasm may require a large dissection. In order to preserve the vascular axis or in the presence of ischemia, the presence of the vascular surgeon reduces the bleeding and allows a more accurate and radical removal of the cancer (Figures 3-5). The majority of patients need chemo and radiotherapy and despite the good immediate results in terms of mortality, during the follow up the appearance of metastases and consequent mortality is not negligible. In a long time follow-up experience of 105 months, the 5-year survival rate reported is $68.4 \%$ [15-16]. For vessels reconstructions the vein is considered ideal substitute for neoplasm and for atherosclerotic obstructive pathology.

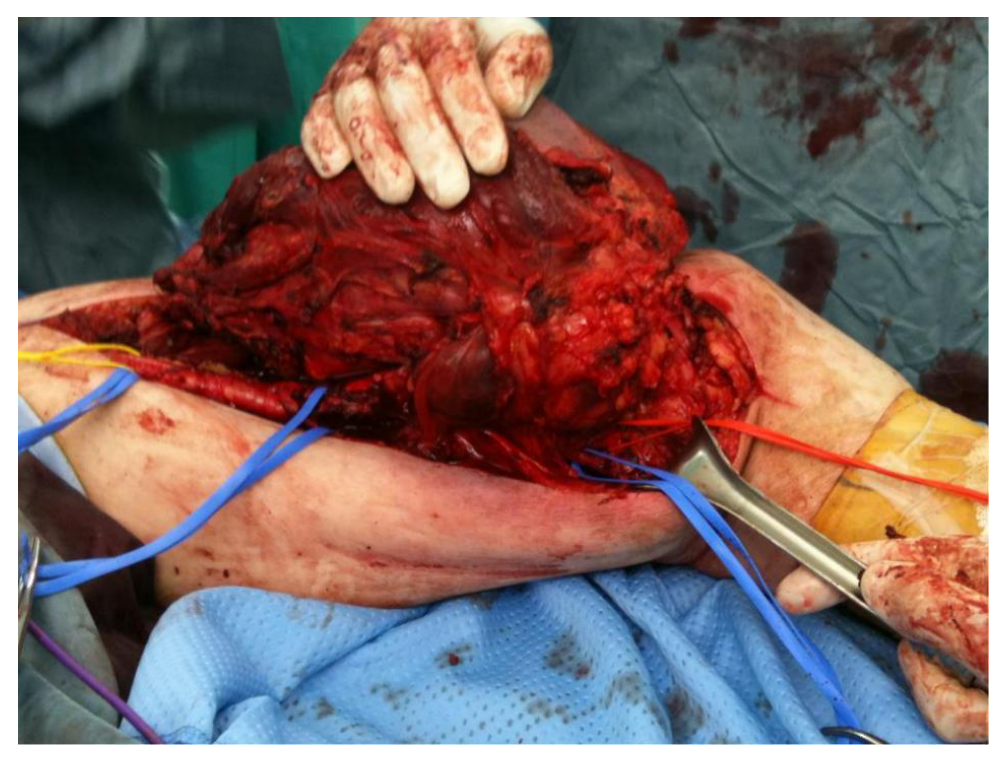

Figure 3: Intraoperative image with preparation of the femoral vessels and isolation of the neoplasm. The vessels were isolated and found in it in yellow and red superficial femoral artery; superficial femoral vein in blue.

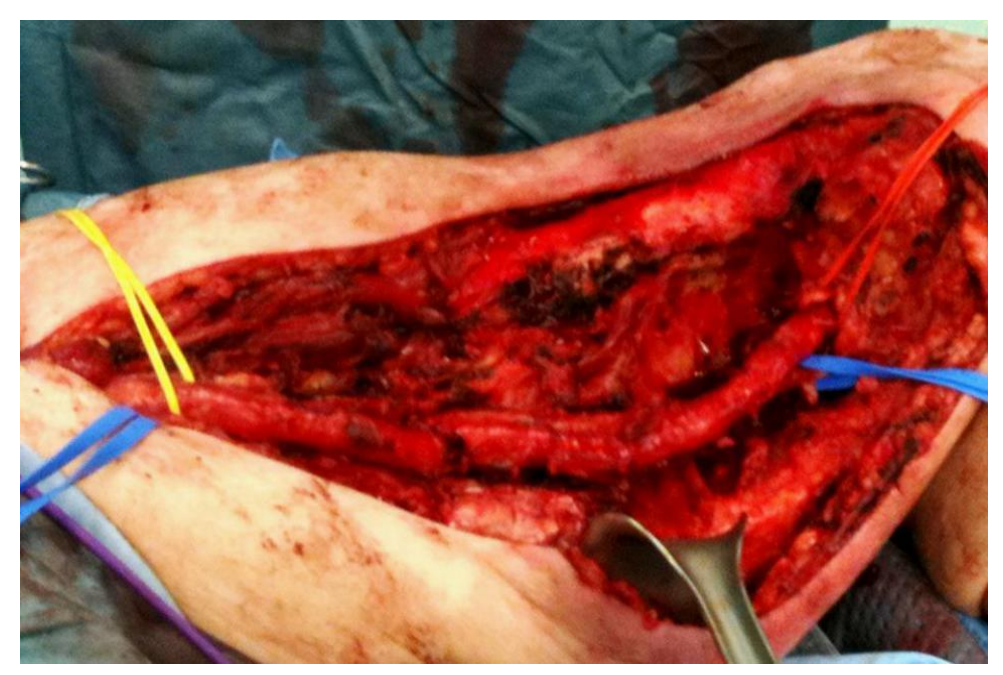

Figure 4: Intraoperative image with the femoral vessels after the sarcoma removal. 


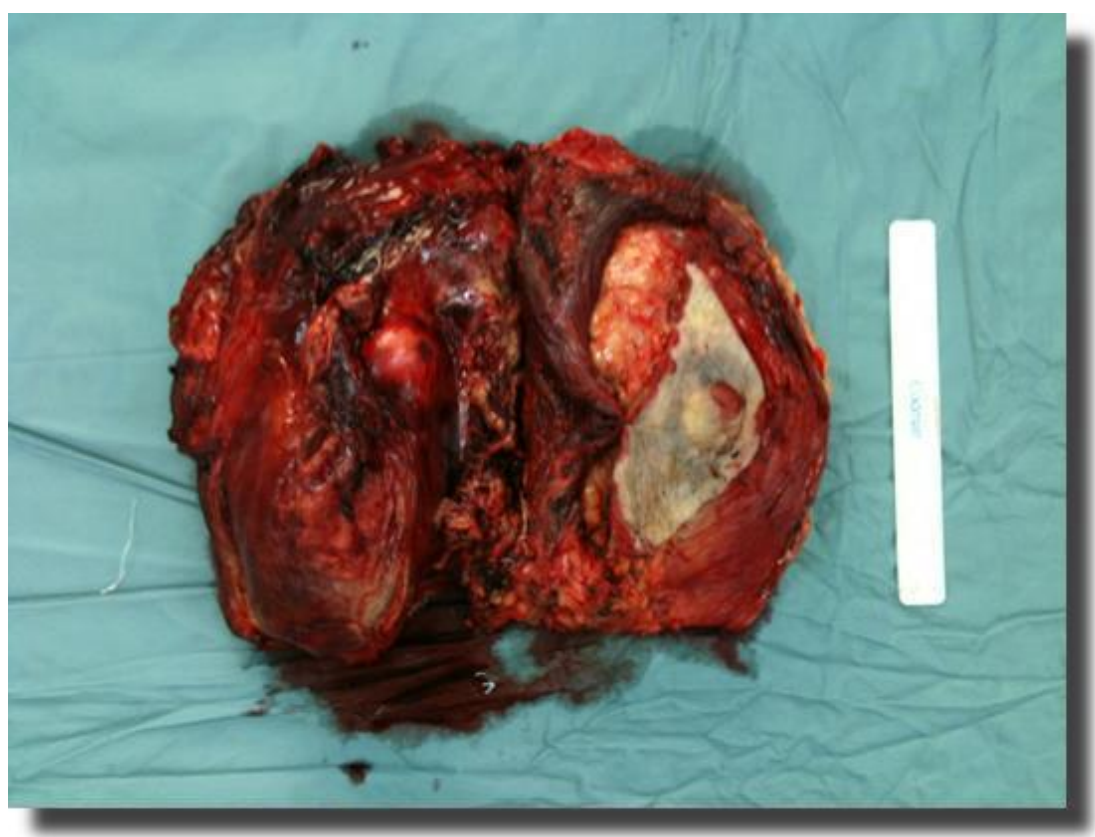

Figure 5: Intraoperative specimen of sarcoma after the surgical removal.

In our experience, in the case of unavailability of suitable venous autogenic substitutes, good results have also been obtained with synthetic prostheses with comparable rates of patency. It is necessary to consider that these are normal arteries, therefore with greater possibility of patency even at a distance. For venous reconstructions, also in our experience the patency was lower, but with no relevant symptoms (Table 1).

\begin{tabular}{|c|c|c|c|c|c|c|c|c|}
\hline Author & Period & Pts & $\begin{array}{l}\text { Mean } \\
\text { Follow up } \\
\text { (months) }\end{array}$ & $\begin{array}{l}\text { Artery/vein } \\
\text { involvment }\end{array}$ & $\begin{array}{l}\text { Artery } \\
\text { Patency at } 30 \\
\text { day \% }\end{array}$ & $\begin{array}{l}\text { Local } \\
\text { Infection }\end{array}$ & Mortality & $\begin{array}{l}\text { Morbidity } \\
\text { (pts/ \%) }\end{array}$ \\
\hline Karakousis & 1991-1996 & 21 & 60 & $\begin{array}{ll}7 / 21 & 33.0 \%\end{array}$ & 100 & $5 \quad 24 \%$ & 0 & not reported \\
\hline McKay & $2002-2005$ & 7 & 20.2 & $7 / 7 \quad 100 \%$ & 100 & 0 & 0 & $5 / 7 \quad 78 \%$ \\
\hline Song & 2003-2008 & 14 & 36 & $7 / 14 \quad 50.0 \%$ & 83 & 0 & 0 & not reported \\
\hline Akgul T & $2004-2007$ & 507 & 39 & $17 / 507 \quad 3.3 \%$ & 82.4 & $5 \quad 29.4 \%$ & & not reported \\
\hline Emory & 1997-2009 & 10 & 48 & $10 / 10 \quad 100 \%$ & 90 & 0 & 0 & $6 / 10 \quad 60 \%$ \\
\hline Muramatsu & $1995-2010$ & 15 & 71.8 & $15 / 15 \quad 100 \%$ & 93.3 & $16 \%$ & 0 & $9 / 15 \quad 60 \%$ \\
\hline Davis & $2005-2013$ & 153 & 12 & $9 / 153 \quad 16.9 \%$ & 89.9 & 0 & 0 & $12 / 15 \quad 37.8 \%$ \\
\hline Cetinkaya & 2002-2014 & 13 & 80.6 & $13 / 13 \quad 100 \%$ & 84.6 & $323.1 \%$ & 0 & $50 \%$ \\
\hline $\begin{array}{l}\text { Our } \\
\text { experience }\end{array}$ & 2011-2014 & 30 & 19.4 & $46.0 \%$ & 100 & 0 & 0 & $15.3 \%$ \\
\hline
\end{tabular}

Table 1: Comparison. 


\section{Conclusion}

Major vessel involvement or injuries induced during cancer surgery may cause serious bleeding. The vascular surgeons in a multidisciplinary approach, plans the exact surgical view through the avascular plane, far from neoplasm and may prevent or minimize these complications. A long-term evaluation is needed to determine the implications at distance in patients undergoing radical resection of cancer. The necessity of vascular surgery skills is rare but essential for limb sparing. The mortality gap depends by the prognosis of neoplasm.

\section{Acknowledgements}

The authors thank the team of Oncological Orthopedics of IFO and Orthopedic and Traumatology A and B Unit of the Tor Vergata Policlinico, for their collaboration.

\section{Conflict of Interest}

The authors declare that they have no conflict of interest.

\section{References}

1. Tunn PU, Kettelhack C, Durr HR. Standardized approach to the treatment of adult soft tissue sarcoma of the extremity. In: Tunn P-U Treatment of Bone and Soft Tissue sarcomas Berlin: Springer (2009): 211-228.

2. Ghosh J, Bhowmick A, Baguneid M. Oncovascular surgery. The journal of cancer surgery EJSO 37 (2011): 1017-1024.

3. Fortner JG, Kim DK, Shiu MH. Malignant Tumors of the Lower Extremity. Limb-Preserving Vascular Surgery for Arch Surg 112 (1977): 391-394.

4. Han A, Ahn S, Min SK. Oncovascular Surgery: Essential Role of Vascular Surgeons in Cancer Surgery. Vasc Specialist Int 35 (2019): 60-69.

5. Schwarzbach MH, Hormann Y, Hinz U, et al. Results of limb-sparing surgery with vascular replacement for soft tissue sarcoma in the lower extremity. J Vasc Surg 42 (2005): 88-97.

6. Tsukushi S, Nishida Y, Sugiura H, et al. Results of limb-salvage surgery with vascular reconstruction for soft tissue sarcoma in the lower extremity: comparison between only arterial and arterovenous reconstruction. J Surg Oncol 97 (2008): 216-220.

7. Adelani MA, Holt GE, Dittus RS, et al. Revascularization after segmental resection of lower extremity soft tissue sarcomas. J Surg Oncol 95 (2007): 455-460.

8. Nishinari K, Wolosker N, Yazbek G, et al. Venous reconstructions in lower limbs associated with resection of malignancies. J Vasc Surg 44 (2006): 1046-1050.

9. Song TK, Harris Jr EJ, Raghavan S, et al. Major blood vessel reconstruction during sarcoma surgery. Arch Surg 144 (2009): 817-822.

10. McKay A, Motamedi M, Temple W, et al. Vascular reconstruction with the superficial femoral vein following major oncologic resection. J Surg Oncol 96 (2007): 151-159. 
11. Muramatsu K, Ihara K, Miyoshi T, et al. Clinical outcome of limb-salvage surgery after wide resectio of sarcoma and femoral vessel reconstruction. Ann Vasc Surg 25 (2011): 1070-1077.

12. Akgul T, Sormaz IC, Aksoy M, et al. Results and functional outcomes of en-bloc resection and vascular reconstruction in extremity musculoskeletal tumors. Acta Orthop Traumatol Turc 52 (2018): 409-441.

13. Laura A Davis, Firas Dandachli, Robert Turcotte, et al. Limb-sparing surgery with vascular reconstruction for malignant lower extremity soft tissue sarcoma. Journal of Vascular Surgery January 65 (2017): 151156.

14. Han SM. Oncovascular Surgery: There Would Be No Such Thing without Vascular Surgeons. Vasc Specialist Int 35 (2019): 53-54.

15. Cetinkaya OA, Celik SU, Kalem M, et al. Clinical Characteristics and Surgical Outcomes of Limb-Sparing Surgery with Vascular Reconstruction for Soft Tissue Sarcomas. Ann Vasc Surg 56 (2019): 73-80.

16. Radaelli S, Fiore M, Colombo C, et al. Vascular resection en-bloc with tumor removal and graft reconstruction is safe and effective in soft tissue sarcoma (STS) of the extremities and retroperitoneum. Surg Oncol 25 (2016): 125-131.

Citation: Ascoli Marchetti A, Di Giulio L, Citoni G, Ippoliti A. Oncovascular Surgery-The Multidisciplinary Approach: Surgical Resection of the Musculoskeletal Tumor and Associated Revascularization. Journal of Surgery and Research 2 (2019): 147-153. 